\title{
Homogeneous Bubble Nucleation driven by local hot spots: a Molecular Dynamics Study
}

\author{
Zun-Jing Wang* \\ FOM Institute for Atomic and Molecular Physics, \\ Kruislaan 407, 1098 SJ Amsterdam, The Netherlands. \\ Department of Physics, Carnegie Mellon University, \\ 5000 Forbes Avenue, Pittsburgh, PA 15213. \\ Chantal Valeriani ${ }^{\dagger}$ \\ FOM Institute for Atomic and Molecular Physics, \\ Kruislaan 407, 1098 SJ Amsterdam, The Netherlands. \\ School of Physics, James Clerk Maxwell Building, \\ Kings Buildings, University of Edinburgh, \\ Mayfield road, EH9 3JZ, Edinburgh, United Kingdom. \\ Daan Frenkel ${ }^{\ddagger}$ \\ FOM Institute for Atomic and Molecular Physics, \\ Kruislaan 407, 1098 SJ Amsterdam, The Netherlands. \\ Department of Chemistry, University of Cambridge, \\ Lensfield Road, CB2 1EW, Cambridge, United Kingdom.
}

(Dated: October 30, 2018)

\footnotetext{
* electronic address: zunjingw@andrew.cmu.edu

$\dagger$ electronic address: cvaleria@ph.ed.ac.uk

$\ddagger$ electronic address: df246@cam.ac.uk
} 


\begin{abstract}
We report a Molecular Dynamics study of homogenous bubble nucleation in a Lennard-Jones fluid. The rate of bubble nucleation is estimated using forward-flux sampling (FFS). We find that cavitation starts with compact bubbles rather than with ramified structures as had been suggested by Shen and Debenedetti ( J. Chem. Phys. 111, 3581 (1999)). Our estimate of the bubblenucleation rate is higher than predicted on the basis of Classical Nucleation Theory (CNT). Our simulations show that local temperature fluctuations correlate strongly with subsequent bubble formation - this mechanism is not taken into account in CNT.
\end{abstract}




\section{INTRODUCTION}

Anyone who has ever sprinkled water droplets into a pan with hot frying oil is familiar with the phenomenon of explosive boiling: the liquid water will be heated far above its equilibrium boiling point before the vapor phase nucleates suddenly and violently. As this example illustrates, explosive boiling is very common, and usually undesirable in practical situations. Bubble nucleation is relevant in many different contexts: in addition to explosive boiling $\frac{1}{1}$, it plays a role in phenomena as diverse as cavitation erosion ${ }^{2}$ and sonochemistry ${ }^{\frac{3}{}}$, and it affects the design of high-efficiency heat exchangers ${ }^{4}$. In spite of the practical relevance of these phenomena, the mechanism by which the vapor phase nucleates from a homogeneous, super-heated liquid is still under debate.

The standard theory used to describe the bubble nucleation phenomenon is the so-called Classical Nucleation Theory (CNT). CNT is commonly used to predict the rate of nucleation and estimate the height of the free-energy barrier $+5,6,7,9$ CNT assumes that the growing droplet of the stable phase within the metastable one is characterized by its bulk properties, it is incompressible, and it has a spherical shape. Moreover, the liquid-vapor interfacial free-energy does not depend on the curvature of the droplet, condition that is satisfied at the surface tension, that coincides with the equimolar dividing surface in case of absence of Gibbs absorption.

From an experimental point of view, CNT is in general used to predict the nucleation rate, both in bubble nucleation and in vapor condensation experiments. Early measurements of vapor condensation performed by Blander and Katz $\stackrel{10}{ }$ turned out to be consistent with the CNT predictions $9,11,12$. However, more recent measurements using a variety of different

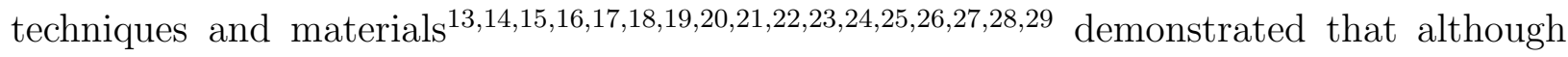
CNT correctly predicts the isothermal supersaturation-dependence of the bubble-nucleation rate, it fails to predict the correct temperature dependence: CNT predicts nucleation rates that are too low at the low temperatures and too large at high temperatures (even allowing for the fact that error bars may span several orders of magnitude). To our knowledge, there exist no experimental techniques that can directly probe the rate-limiting step in the nucleation process, namely to formation of "critical" bubbles.

In an attempt to improve upon the predictions of CNT, Zeng and Oxtoby ${ }^{30}$ have used 
Density-Functional Theory (DFT) to arrive at an estimate of bubble nucleation rate. In particular, Zeng and Oxtoby studied the liquid-to-vapor phase transition in a LennardJones fluid and found that CNT underestimated the nucleation rate by more than 15 orders of magnitude. In order to explain the discrepancy between the DFT predictions and the CNT estimates, Oxtoby and co-workers $\frac{31}{32}$ pointed out that CNT neglects all curvature corrections to the surface free-energy of a droplet. Another curious feature of CNT that was already pointed out by Cahn and Hilliard ${ }^{33,34}$ and discussed in some detail by Oxtoby and Evans $^{35}$ is the fact that this theory predicts a finite nucleation barrier at the point where the metastable liquid undergoes spinodal decomposition - this topic is still the subject of much debate $.^{36}, 37$ Delale et al.$\frac{38}{}$ proposed a phenomenological estimate of the minimum work of bubble formation, using experimental data on super-heating. Using this approach, ref. 38 estimated the steady-state bubble nucleation rate and found that, as with the CNT estimates, there was a large discrepancy between the predicted computed and measured bubble-nucleation rates.

In 1999, Shen and Debenedetti ${ }^{39}$ reported a Monte Carlo (MC) study to estimate the free-energy barrier for bubble nucleation in a monatomic fluid of particles interacting via a truncated Lennard-Jones potential. These authors computed the free-energy barrier using Umbrella Sampling $\underline{40,41}$, and monitored the progress of bubble formation using the total density of the system as an order parameter. A geometric analysis of the resulting void structures in the superheated liquid revealed that the formation of the vapor phase proceeded via the formation of ramified, system-spanning void structures. This observation disagrees qualitatively with the predictions of CNT and DFT, both of which assume that the critical nucleus is a compact, spherical bubble. It should however be noted that ref. 39 studied a system very close to the point where the system is expected to undergo spinodal decomposition.

The aim of the present paper is to study the kinetics of bubble nucleation under conditions similar to those studied in ref. 39. In order to study the time evolution of bubble nucleation, we must use Molecular Dynamics (MD) simulations and because it is then better to use an interaction potential such that the forces vanish continuously at the cut off, we could not use exactly the same model as Shen and Debenedetti. Rather, we studied a system of particles interacting via a truncated, force-shifted Lennard-Jones potential. However, 
as we shall argue below, we study thermodynamic conditions that are comparable. Using Forward-Flux Sampling (FFS) $\underline{42,43}$, we compute the bubble nucleation rate and compare our numerical estimate with the prediction of CNT. FFS is well suited to simulate nucleation processes because it does not assume that the nucleation process is slow compared to the time it takes all other degrees of freedom to equilibrate. The latter assumption is implicit in the Umbrella Sampling scheme.

The rest of this paper is organized as follows: we first describe the simulations techniques used in our work, then we present our results and discuss the implications.

\section{SIMULATION METHODS}

In order to probe the mechanism of bubble nucleation, we simulated a system consisting of 3375 particles interacting via a truncated and force-shifted (TSF) Lennard-Jones (LJ) pair potential:

$$
v_{T S F}(r)=v_{L J}(r)-v_{L J}\left(r_{c}\right)-\left|\frac{d v_{L J}}{d r}\right|_{r_{c}}\left(r-r_{c}\right) .
$$

The TSF-LJ potential is a modification of the 12-6 Lennard-Jones potential. The second and third terms on the right hand side of Eq. 1 have been chosen such that both the potential and its first derivative vanish continuously at $r_{c}=2.5 \sigma$ (where $\sigma$ is the particle diameter). The reason why we use the TSF-LJ rather than the full LJ potential is that during nucleation the system becomes inhomogeneous. Under those conditions it is much simpler to use a finite-ranged potential than an interaction with an $r^{-6}$-tail.

In what follows, we use reduced units: all physical variables are expressed in terms of the Lennard-Jones units $\sigma, m, \varepsilon$, where $\sigma$ is a measure for the diameter of a Lennard-Jones particle, $m$ denotes its mass and $\varepsilon$ is the depth of the attractive well of the (untruncated) LJ potential.

We start our simulations by equilibrating the system in an NPT ensemble by means of MD simulations with a Nosé-Hoover thermostat $\underline{44}, \underline{45}, \underline{46}$ and an Andersen barostat $\underline{47}$ (Appendix A). In order to study the bubble nucleation phenomenon, we use MD rather than MC simulations, because standard MC algorithms assume that the system is always locally in thermal equilibrium - an assumption that may not be justified in the present case. In all simulations, we adopt cubic periodic boundary conditions. The equations of motion are in- 
tegrated using a leap-frog algorithm ${ }^{48}$ with an integration time step of $\Delta t^{*}=0.00046$, which corresponds to a time step of about 1 femto-second (using the Lennard-Jones parameters for Argon). In order to validate the MD code used in the present work, we verified that it could successfully reproduce the liquid-vapor coexistence curve of the TSF-LJ model that was computed by Errington et al. $\underline{\underline{49}}$ (Appendix B).

In an NPT ensemble the simulation box adjusts itself in order to keep both the temperature and the pressure of the system constant. When a system undergoes a liquid-to-vapor during an NPT simulation, the simulation box tends to expand dramatically: in our simulation runs we limit such "explosions" by interrupting the runs before the liquid phase has evaporated completely.

In order to study bubble nucleation, the system should be large enough to accommodate a "critical" bubble (i.e. a bubble that is equally likely to grow or shrink). On the basis of CNT we expect that the size of the critical bubble is inversely proportional to the degree of super-saturation. If we carry out simulations at a low supersaturation, the critical bubble size can become very large and this would necessitate the use of very large simulation boxes (containing a large number of particles). On the other hand, if we choose the supersaturation too large, the liquid-vapor phase transformation will not proceed via nucleation and growth but through spinodal decomposition. These two constraints put rather narrow limits on the values of supersaturation that can conveniently be studied.

In order to obtain a rough estimate of the critical bubble size, we make the CNT assumption that the chemical potential of the vapor inside the critical bubble is the same as that of the liquid outside. If we use the fact that the molar volume of the vapor phase is much larger than that of the liquid phase, this implies that pressure inside the critical bubble is the same as the equilibrium vapor pressure at the imposed temperature. The radius of the critical bubble can then be approximated as:

$$
r_{c r i t}=\frac{2 \gamma}{P_{v p}(T)-P}
$$

where $\gamma$ is the liquid-vapor surface tension, $P$ is the imposed external pressure of the liquid and $P_{v p}(T)$ is the equilibrium vapor pressure at the temperature $T$.

Shen and Debenedetti ${ }^{39}$ studied bubble formation in the truncated Lennard-Jones system at a saturated vapor pressure of $P^{*}=0.046$ and various degrees of super-heating, defined 
as $S \equiv\left(T^{*}-T_{\text {sat }}\right) / T_{\text {sat }}$, where $T_{\text {sat }}$ the vapor-liquid coexistence temperature at the given pressure). For $S=9 \%$, CNT predicts a free-energy barrier of $37 k_{B} T$ for bubble nucleation $\underline{39}$ In the present study, we do not use exactly the same model as ref. 39, but we do use the same degree of super-heating $(S=9 \%)$. Although the FTS-LJ model is not identical to the model used in ref. 39, our simulations were carried out at approximately the same distance from the critical point $\left(T^{*} / T_{c}^{*}=0.914\right)$. For this reduced temperature and supersaturation, we expect that a system of 3375 particles can accommodate a critical bubble. To summarize: we carried out simulations at a temperature $T^{*}=0.855$ and a reduced pressure $P^{*}=0.026$. The coexistence temperature at this reduced pressure is $T_{s a t}^{*}=0.785$. The critical temperature of the TSF-LJ model was estimated by Errington et al. $\underline{\underline{49}}$ to be $T_{c}^{*}=0.935$. At coexistence $\left(T_{s a t}^{*}=0.785, P_{s a t}^{*}=0.026\right)$, the number densities of the coexisting liquid and vapor are,

respectively, $\rho_{l, \text { coex }}^{*}=0.668$ and $\rho_{v, \text { coex }}^{*}=0.043$. The number density of the superheated liquid at $T^{*}=0.855$ and $P^{*}=0.026$ is $\rho_{l}^{*}=0.580$.

\subsection{Liquid-vapor surface tension}

In order to compare our numerical estimate for the nucleation rate with the prediction based on CNT, we need to know the value of the surface tension $\gamma$. As both the critical temperature and the surface tension are sensitive to the long-range part of the intermolecular potential, it is important to have a reliable estimate of $\gamma$ for the TSF-LJ potential truncated at $r_{c}=2.5 \sigma$ for the state point studied in the simulations.

To estimate $\gamma$, we have performed MD simulations of the TSF-LJ liquid-vapor coexistent systems at $T^{*}=0.785\left(P^{*}=0.026\right)$ and at $T^{*}=0.855\left(P^{*}=0.046\right)$ separately. We obtain the surface tensions of the planar interfaces: $\gamma^{*}=0.204 \pm 0.007$ at $T^{*}=0.785$, and $\gamma^{*}=0.098 \pm 0.008$ at $T^{*}=0.855$ (Appendix C). Lutsko has used DFT to estimate the liquid-vapor surface tension of the TSF-LJ system at the same state points and obtained $\gamma_{D F T}^{*}=0.198$ at $T^{*}=0.785$, and $\gamma_{D F T}^{*}=0.119$ at $T^{*}=0.855 . \underline{50.51}$ These values for the surface tension are very similar to the ones for the model studied in ref. 39. In view of the statistical errors in the simulation data, the agreement between the DFT estimates for $\gamma^{*}$ and the simulation results is quite good. We did not compute the surface tension of a super-heated system at $T^{*}=0.855$ and $P^{*}=0.026$ because, under this thermodynamic 
condition a flat interface is not thermodynamically stable.

\subsection{The order parameter}

Even in the stable liquid phase, spontaneous density fluctuations occur. In a superheated liquid, some of these local density fluctuations may grow to nucleate a bubble of the stable vapor phase. In order to follow the nucleation process we need an "order parameter" that provides a quantitative measure of the degree of transformation from the liquid to the vapor phase. In the present simulations we used the volume of the biggest bubble as an order parameter. This is a local order parameter that is sensitive to the formation of large, connected void spaces. The present definition of the order parameter is different from the one used by Shen and Debenedetti, who used the overall density of the system as a global order parameter. We will return later to this difference in the choice of order parameters.

We used the following procedure to identify the largest connected low-density region in the superheated liquid:

1. Following the method used in ref. 52, we construct a three-dimensional grid with cell mesh of $(0.5 \sigma)^{3}$ inside the simulation box ( $\sigma$ is the LJ diameter).

2. As in ref. 53, we use Stillinger's cluster criterion ${ }^{54}$ to identify the nearest neighbors of each particle: according to our definition, two particles are neighbors if their distance is less than a cut-off $r_{C}=1.6 \sigma$, corresponding to the first minimum of the radial distribution function of the liquid at the same thermodynamic conditions. Using this criterion, we compute the probability distribution of each particle's neighbors in both the liquid and the vapor phases.

3. We then identify a particle as liquid-like if it has more than five neighbors. We used this particular threshold, as essentially all particles in the homogeneous vapor phase have fewer neighbors and almost all particles in a homogeneous liquid phase have more (see Figure 1).

4. We define a spherical volume with radius $r_{C}$ around every liquid-like particle: all grid cells which are inside this spherical volume are labelled as liquid-like. The remaining grid cells are labelled vapor-like. 
5. Finally, we perform a cluster-analysis on the vapor-like grid cells: a set of connected, vapor-like cells defines a bubble. We then select the largest of those and define this as the largest bubble. The local order parameter is the volume $W_{b}$ of the largest bubble expressed in units of $\sigma^{3}$ (for practical reasons, we multiply the bubble volume by 10 and express it as an integer in FFS computations).

\subsection{Forward Flux Sampling with Molecular Dynamics}

FFS is a technique that allows us to track the pathways and flux of a rare phase transition between two regions of the phase space, the liquid $\left(L_{l i q}\right)$ and the $\operatorname{vapor}\left(V_{\text {vap }}\right)$. As bubble nucleation is a rare-event phenomenon, we incorporated FFS technique $\underline{42}, \underline{43}$ in the MD simulations of the super-heated fluid. There are specific complications that arise when FFS is combined with MD and, to our knowledge, this had not been attempted before.

Once we have defined a parameter $\lambda$ that measures the progress of the nucleation process, we can define a value of $\lambda$, denoted as $\lambda_{0}$, that define the boundary of the metastable liquid region. By this we mean a value of $\lambda$ that is large enough to ensure that the overwhelming majority of the states of the meta-stable liquid have a lower value of $\lambda$, yet small enough to ensure that occasional a spontaneous fluctuation will cross $\lambda_{0}$. We stress that the choice of $\lambda_{0}$ is to some extent arbitrary and that our results do not depend on the precise choice. Similarly, we define a maximal value of $\lambda$, denoted as $\lambda_{n}$, such that almost all trajectories that reach this value of $\lambda$ will continue in the direction of progressive evaporation. We denote the domain of the meta-stable liquid $\left(\lambda<\lambda_{0}\right)$ by $L_{\text {liq }}$ and that of the vapor $\left(\lambda>\lambda_{n}\right)$ by $V_{\text {vap }}$.

As the transition from $L_{l i q}$ to $V_{v a p}$ is rare, the system will initially spend most of its time in the $L_{l i q}$-basin with $\lambda<\lambda_{0}$. The key idea of FFS is to use a series of non-intersecting hypersurfaces (interfaces) in phase space, each one identified by a value of the order parameter $\lambda>\lambda_{0}$, to drive the system from $L_{l i q}$ to $V_{v a p}$ in a ratchet-like manner.

The first step in the simulation is to compute $\Phi$, the flux of trajectories that cross $\lambda_{0}$ in the direction of increasing bubble size, per unit time and per unit volume. We store the configurations of the system at the time that a "forward" trajectory crosses $\lambda_{0}$. Once a set of such configurations have been collected at $\lambda_{0}$, we fire off stochastic trajectories that start 
from $\lambda_{0}$ and either reach the next interface $\lambda_{1}$ or fall all the way back to the initial basin $L_{l i q}\left(\lambda_{0}\right)$. We then compute $P\left(\lambda_{0} \mid \lambda_{1}\right)$, the probability that a trajectory that had crossed $\lambda_{0}$ continues to $\lambda_{1}$, rather than return to the initial basin $L_{l i q}$. We collect the configurations at $\lambda_{1}$ where trajectories from $\lambda_{0}$ first cross. Next we use these configurations to start new stochastic trajectories that will either reach $\lambda_{2}$ or fall back to $\lambda_{0}$. In this way we compute $P\left(\lambda_{1} \mid \lambda_{2}\right)$, the probability a trajectory started at $\lambda_{1}$ reaches $\lambda_{2}$ before returning to the initial basin $L_{l i q}$. We then iterate this procedure all the way to the final boundary $\lambda_{n}$. The FFS estimate for the nucleation rate is then expressed as the product of two terms:

$$
R_{L_{l i q} V_{v a p}}=\Phi \cdot \prod_{i=0}^{n-1} P\left(\lambda_{i} \mid \lambda_{i+1}\right)
$$

The above description of the FFS technique makes it clear that there is a problem when combining FFS with MD: FFS that the underlying dynamics is stochastic, such that different trajectories can be generated from the same initial point in phase space. In contrast, standard Newtonian dynamics is deterministic, hence there is only one trajectory emanating from a given point in phase space. In order to combine FFS with MD simulations in an NPT ensemble, we make use of a weakly stochastic variant of the (deterministic) Nosé-Hoover thermostat. To this end, we add a Lowe-Andersen (LA) thermostat to the Nosé-Hoover thermostat ${ }^{55}$. The LA thermostat is a momentum conserving and Galilean invariant analog of the Andersen thermostat: it re-scales the relative velocity between two particles from a Gaussian distribution with a probability $\Delta t^{*} \nu^{*}$, where $\Delta t^{*}$ is the MD time step and $\nu^{*}$ a tunable parameter indicating the strength of the coupling between the system and the thermostat (the smaller the $\nu^{*}$, the weaker the coupling). Therefore the dynamical properties of the system can be tuned by varying $\nu^{*}$. The larger $\nu^{*}$ the more stochastic the dynamics of our system. Of course, we wish to ensure that the stochastic nature of the LA thermostat has only a minor effect on the dynamical properties of the system. As a test, we computed how the value of $\nu^{*}$ affectes the self-diffusion coefficient in the FST-LJ system (see Figure 21). We find that when $\nu^{*} \leq 100$, the diffusion of the system with the LA thermostat is indistinguishable from the one obtained with the deterministic Nosé-Hoover thermostat. In fact, there is little change in the diffusion coefficient as long as $\nu^{*} \leq 1000$. However, when $\nu^{*} \geq 10000$, the LA thermostat clearly affects the dynamics.

In order to balance the added stochasticity to the need of keeping the system's dynamics 
as Newtonian as possible, we choose $\nu^{*}=500$. As Figure 3 shows, the added stochasticity is enough to ensure divergence of the trajectories in phase space during the time it takes to travel from one interface to the next. $\underline{56}$

\section{RESULTS AND DISCUSSION}

To perform an MD-FFS of bubble nucleation in a superheated liquid system at $T^{*}=0.855$ and $P^{*}=0.026$, we locate the boundary of the metastable liquid basin at $W_{b}=25$, and set the interfaces at monotonically increasing values of the order parameter $W_{b}$ : $W_{b, 1}=37$, $W_{b, 2}=57, W_{b, 3}=92, W_{b, 4}=120, W_{b, 5}=180, W_{b, 6}=240, W_{b, 7}=300, W_{b, 8}=400$, $W_{b, 9}=550, W_{b, 10}=700, W_{b, 11}=820, W_{b, 12}=950, W_{b, 13}=1200, W_{b, 14}=1900$. At each interface we store $O\left(10^{2}\right)$ configurations, select 30-50 of these at random and fire $O\left(10^{2}\right)$ trajectories from each of these. At a value of $W_{b} \sim 950$, approximately $50 \%$ of all trajectories continue to the vapor phase. This value of $W_{b}$ then constitutes our operational definition of the critical bubble size. However, we stress that, as $W_{b}$ is not a perfect reaction coordinate, the committor of individual configurations with $W_{b}=950$ may differ from $50 \%$. We have not attempted to optimize the choice of the reaction coordinate to make it coincide more closely with an equi-committor surface. At $W_{b}=3500$, the committor to reach the vapor phase is very nearly equal to one: we use this value of $W_{b}$ as the boundary of the vapor phase. Once simulations have reached this point, they are terminated.

Using FFS, we obtain the following estimate for the bubble nucleation rate at $T^{*}=0.855$ and $P^{*}=0.026: R=9 \times 10^{-15 \pm 1} \sigma^{-3} \tau^{-1}$. Using the Lennard-Jones parameters of Argon, this rate becomes $R=10^{19} \mathrm{~cm}^{-3} \mathrm{~s}^{-1}$. The computed bubble-nucleation rate is much higher than the one measured in early experiments for a number of volatile organic liquids: as an example, the bubble nucleation rate from ref. 10 is $10^{4}-10^{6}$ bubbles $\mathrm{cm}^{-3} \mathrm{~s}^{-1}$ at a temperature about $0.89 T_{c}$ and an ambient pressure $1 \mathrm{~atm}$. 


\subsection{Comparison with Classical Nucleation Theory}

A CNT-based theoretical estimate of the bubble nucleation rate was given by Katz (Eq. 12 in Ref. 10):

$$
R_{C N T}=N\left[\frac{2 \gamma}{\pi m B}\right]^{1 / 2} \exp (-\beta \Delta G)
$$

where the prefactor $\left(N\left[\frac{2 \gamma}{\pi m B}\right]^{1 / 2}\right)$ contains the number density of the liquid $(N)$ and the Zeldovitch factor $\left(\left[\frac{2 \gamma}{\pi m B}\right]^{1 / 2}\right), m$ is the mass of a molecule, $\gamma$ the liquid-vapor surface tension, and $B$ a term that takes into account the mechanical equilibrium of the bubble: in cavitation experiments, $B$ is always equal to one. $\beta \Delta G$ is the free-energy barrier to form the critical bubble, equal to $\frac{16 \pi \gamma^{3}}{3\left[P_{V}-P_{L}\right]^{2}}$ for a spherical bubble.

The liquid-vapor surface tension of a planar interface of the TSF-LJ fluid at $T^{*}=0.855$ is $\gamma^{*}=0.098 \pm 0.008$ from MD simulations. This leads to a free-energy barrier of $\beta \Delta G=$ $47 \pm 11$. Computing the bubble nucleation rate by means of Eq. 4, we find that CNT predicts $R \approx 10^{-22} \sigma^{-3} \tau^{-1}$, with an estimated error of five orders of magnitude in either direction. The CNT prediction is six orders of magnitude less than the simulation results. However, in view of the statistical errors in the data, this discrepancy is only marginal. Using the DFT estimate for the surface tension $\gamma^{*}=0.119$ at $T^{*}=0.855$, we obtain a CNT estimate $R \approx O\left(10^{-36}\right) \sigma^{-3} \tau^{-1}$, which is $21 \pm 1$ orders of magnitude smaller than the MDFFS computation. Our finding is consistent with earlier theoretical studies that found that CNT estimates of bubble-nucleation rates were always lower than the DFT results $30,31,32$. Of course, the DFT estimate of the surface tension at $T^{*}=0.855$ refers to the value for a planar interface at coexistence.

\subsection{Bubble nucleation pathways}

Figure 4 shows a typical example of a computed pathway for bubble nucleation in a super-heated liquid at $T^{*}=0.855$ and $P^{*}=0.026$. As can be seen from Figure 4, the growing bubble is compact, even the critical bubble. In this respect, our findings appear to differ qualitatively from the findings of ref. 39. As the present simulations were carried out at virtually the same distance from the critical point and at the same superheating, it is likely that the difference in the results is due to a difference in the techniques that were 
used to follow the progress of bubble nucleation. In particular, in the present study we made use of a local order parameter (viz. the size of the largest compact bubble), whereas ref. 39 computed the free-energy as a function of a global order parameter (viz. the density of the entire system). These two methods need not probe the same process. As discussed in ref. 57 there are two ways in which a system can accommodate an increase in a global nucleation order parameter. The first is via the growth of a compact nucleus, i.e. the process that we normally associate with nucleation. The second is via the generation of many small nuclei that, together, yield the same value of the global order parameter. If the system is large enough and the surface tension small enough, the second route will be favored for entropic reasons. As the global order parameter increases, the many small nuclei may form a percolating structure, as was indeed observed in ref. 39. The fact that in our FFS simulation we do not observe this scenario but the nucleation of a compact bubble indicates that the latter pathway is kinetically favored.

We stress that the use of an order parameter that is based on the size of the largest bubble would still allow us to observe the formation of ramified clusters if this were the favored route: it would simply show up as sudden changes in the size of the largest bubble as it merges with other (smaller) bubbles. Interestingly, we occasionally do observe such processes, be it on a small scale: we find that sometimes two small bubbles may merge to form a larger bubble - we also observe the converse process where a larger bubble spontaneously breaks up into two smaller bubbles. When this happens the volume $W_{b}$ of the largest bubble changes discontinuously (see Figure 5). However, unlike ref. 39 we find that the resulting bubbles are always compact.

The conclusion must therefore be that FFS would in principle allow for the formation of ramified structures, as observed in ref. 39. The fact that we do not see such structures suggests that this pathway is kinetically unfavorable. Such kinetic effects could not be observed in the equilibrium umbrella-sampling study of ref. 39. There are, however, other examples of nucleation events where the system appears to prefer a higher free-energy route for purely kinetic reasons. ${ }^{58}$ 


\subsection{Local Kinetic Energy of Bubble Nucleus}

There exists and extensive literature on the study of rare events in condensed phases (see, for instance refs. 59,60). In the numerical study of rare events, such as activated steps in chemical reactions or nucleation events, the reaction coordinate is usually constructed in configuration space. In other words: the most common situation is one where the success of a barrier-crossing trajectory does not depend on the initial momenta of the particles involved in the rare event. However, in bubble nucleation the situation may be different. To see this, consider the converse of bubble nucleation, viz. bubble collapse. Bubble collapse tends to proceed sufficiently rapidly that heat cannot be transported away quickly enough to keep the process at constant temperature. Hence, at the end of a bubble collapse the system may be locally very hot. This is the physical origin of sono-luminescence (see, e.g. ref. 61). It is therefore reasonable to assume that the reverse process, bubble nucleation, will preferentially originate in a part of the system that is locally hotter than its surroundings. Such an effect cannot be studied using a technique such as umbrella sampling, as this approach is based on the assumption of local thermal equilibrium. However, FFS makes no such assumptions and therefore allows us to see if local temperature fluctuations affect the propensity for bubble nucleation.

To investigate the relation between local heating and subsequent nucleation, we studied the FFS trajectories of a large number of successful bubble-nucleation events. We stress that the initial conditions in these simulations were not biased: i.e. we did not modify the initial temperature. Once a bubble had successfully nucleated, we traced back the trajectory to the metastable liquid basin and analyzed the local temperature along this trajectory. To improve the statistics, we did this for a number of other successful trajectories that started from the same initial conditions. There are several ways to define the relevant local temperature. We used the following procedure: we identified all particles in the vapor phase inside the largest bubble at each interface along FFS trajectory and then tracked the kinetic energy of those particles from the basin to the end of FFS trajectories during the bubble nucleation process. As an example, we show the ensemble of the configurations collected at each interface from the successful pathways at $T^{*}=0.855$ and $P^{*}=0.026\left(T_{\text {sat }}^{*}=0.785\right)$ in Table 1.

In order to see if temperature has an effect on the propensity for bubble nucleation we 
compared the local temperatures of bubbles that were committed to grow with that of all bubbles that have reached the same size. We can then compare these two temperatures with the temperature of the remainder of the system at the same point on the FFS trajectory. Figure 6 shows that the bulk liquid temperature of the total system computed with the $N_{i}^{s}$ collected MD snapshots at each interface $i$ is effectively constant and equal to the imposed temperature of thermostat.

Figure [6 shows that a successful nucleation is likely to have started in a region where the local temperature is higher than the temperature of the bulk liquid. In other words, a growing bubble is, at least initially, hotter than the surrounding liquid. From interface 6, the local temperatures of the largest bubbles is not significantly different from the bulk temperature. By analyzing the overall volume of the system, we find that it barely changes from interfaces $0 \sim 4$, whereas it starts increasing from interface 5 (see Figure 7). Interestingly, the system expansion starts at the point where the bubble has cooled to the temperature of the surroundings.

Between interface 5 and the critical (iso-committor) surface 12, there appears to be a difference between bubbles that continue to grow and those that shrink. On average, the temperature of growing bubbles appears lower than the ambient temperature, whilst is not significantly different from ambient. Note, however, the statistical errors are large: individual points may not conform to this general trend. It should be noted that the effect of local temperature on nucleation may, in practice, be somewhat larger than indicated here because the system that we study is always weakly thermostatted. Hence, to return to the example of the collapsing bubble, the final temperature reached on collapse would be somewhat less in our simulations than in reality.

The main conclusion of the present section is that local temperature fluctuation play an important role in bubble nucleation. A description such as CNT that is based on the assumption of local thermal equilibrium cannot capture this phenomenon adequately. In the wider context of activated processes in condensed media it is interesting to find an example of a rare event where the kinetic energy appears to be a relevant quantity to determine the propensity for the subsequent barrier crossing. Such an observation would not be surprising for barrier crossings that are largely ballistic, but it has, to our knowledge, not been observed in the case of more diffusive barrier crossings. We stress, however, that 
the effect of local temperature fluctuations on bubble nucleation is not surprising: this phenomenon is responsible for the operation of the bubble chamber to detect elementary particles.

\section{CONCLUSIONS}

Combining Molecular Dynamics simulations with Forward-Flux Sampling, we have studied the kinetics of bubble nucleation in a truncated force-shifted Lennard-Jones liquid. In our simulations, we used the volume of the largest bubble as the order parameter to follow the phase transition. Using FFS, we computed the bubble nucleation rate and compared it with the one predicted by CNT. The simulated nucleation rates appear significantly larger than those predicted by CNT. However, the CNT estimates are subject to considerable uncertainty, as small statistical errors in the calculated value of the surface tension have huge effects on the estimates for the nucleation rate. We note that the suggestion that CNT underestimates bubble nucleation rates also follows from a comparison of predictions based on $\mathrm{CNT}$ and DFT $\underline{30}$. Analysis of the nucleation pathway indicates that nucleation takes place via the formation of compact bubbles. These bubbles undergo shape fluctuations but are mostly compact and fairly spherical. We do not find evidence for the existence of ramified and percolating void structures ${ }^{39}$. We argue that the difference between our findings and those of ref. 39 is not so much due to a difference in the models studied but to the use of a global order parameter in ref. 39. We find that local temperature fluctuations are important for the propensity of bubble nucleation. At the beginning of nucleation event, the incipient bubble is always significantly hotter than the surrounding bulk liquid. When the total volume of the system starts increasing, the bubbles cools down to ambient temperature.

\section{Acknowledgments}

The authors thank J. R. Errington for sharing his data of the truncated force-shifted phase diagram, J. Lutsko for providing us the DFT estimate of the liquid-vapor surface tension, and J. van Meel for a careful reading of the manuscript. The work of the FOM Institute is part of the research program of the Stichting voor Fundamenteel Onderzoek 
der Materie (FOM), which is financially supported by the Nederlandse Organisatie voor Wetenschappelijk Onderzoek (NWO). 


\section{Appendix A: Details on the used thermostat and barostat}

In our simulations, we use a thermostat and a barostat to keep the thermodynamic conditions of the system constant. However, we wish to work under conditions where the temperature and pressure control do not affect the dynamics of the system appreciably on the timescale for molecular velocity fluctuations. To select reasonable relaxation times for both the thermostat and the barostat, we performed several trial runs at different temperatures and pressures before selecting $\tau_{T}^{*}=0.093$ for the thermostat and $\tau_{P}^{*}=1.390$ for the barostat. Figure A-1 illustrates the (kinetic) temperature fluctuations in the fluid about a value of $\left\langle T^{*}\right\rangle=0.900$ and the (virial) pressure fluctuations in the same fluid about a value of $\left\langle P^{*}\right\rangle=$ 0.064

The mass of the "piston" in the barostat was chosen such that the timescale for volume fluctuations corresponded to the time it takes a sound wave to traverse the simulation box (approximately $1 \mathrm{ps}) \underline{\underline{47}}$

\section{Appendix B: TSF-LJ liquid-vapor phase diagram}

Errington et al. ${ }^{49}$ computed the liquid-vapor phase diagram of the TSF-LJ by means of MC simulations. In fig. B-1 we show Errington's data for the TSF-Lennard Jones $\left(r_{c}=2.5 \sigma\right)$. For the sake of comparison, we also reproduce the liquid-vapor phase diagram data of the full Lennard Jones calculated by Potoff et al. $\frac{62}{}$ and by Smit $\underline{63}$ and the corresponding results for the truncated and shifted Lennard-Jones at $r_{c}=2.5 \sigma$ calculated by Smit $\underline{63}$ and by Trokhymchuk et al. $\underline{\underline{64}}$. In the same figure, we also plot our own simulation data for the TSF-LJ potential. We find excellent agreement between our own simulation results and the data of ref. 49 .

As was already pointed out by Smit $\underline{63}$, truncating and shifting the potential has a large effect on the location of the critical temperature. Figure B-1 illustrates this observation.

\section{Appendix C: liquid-vapor surface tension computed with MD simulations}

To compute the liquid-vapor surface tension, we start by preparing a half-liquid-halfvapor system with 16000 particles. After having equilibrated a liquid by means of an NPT 
simulation at the coexistence temperature and pressure $\left(T^{*}=0.785, P^{*}=0.026\right.$ or $T^{*}=$ $\left.0.855, P^{*}=0.046\right)$, we prepare a liquid-vapor interface by evaporating half of the system. The thickness of the liquid slab and the size of simulation box are chosen sufficiently large to make finite-size effects unlikely. Once the system of coexisting liquid and vapor has reached thermodynamic equilibrium, we calculate the liquid-vapor surface tension in an NVT ensemble (see ref. 65). We compute the density profile $\rho(z)$, and the normal and tangential pressure profiles, $P_{N}(z)$ and $P_{T}(z)$ along the $z$ direction perpendicular with respect to the liquid-vapor dividing interface (see Figure C-1), and use the Irving-Kirkwood approach to

compute the pressure tensor $\underline{\underline{66}}$. The normal component of the pressure tensor is expressed as

$$
P_{N}(z)=\langle\rho(z)\rangle k_{B} T-\frac{1}{A}\left\langle\sum_{i} \sum_{j>i} \frac{z_{i j}^{2}}{r_{i j}} \frac{d U\left(r_{i j}\right)}{d r_{i j}} \frac{1}{\left|z_{i j}\right|} \times \theta\left(\frac{z-z_{i}}{z_{j}-z_{i}}\right) \theta\left(\frac{z_{j}-z}{z_{j}-z_{i}}\right)\right\rangle,
$$

whereas the tangential component is given by

$$
P_{T}(z)=\langle\rho(z)\rangle k_{B} T-\frac{1}{A}\left\langle\sum_{i} \sum_{j>i} \frac{x_{i j}^{2}+y_{i j}^{2}}{2 r_{i j}} \frac{d U\left(r_{i j}\right)}{d r_{i j}} \frac{1}{\left|z_{i j}\right|} \times \theta\left(\frac{z-z_{i}}{z_{j}-z_{i}}\right) \theta\left(\frac{z_{j}-z}{z_{j}-z_{i}}\right)\right\rangle,
$$

where $\rho(z)$ denotes the density profile along the $\mathrm{z}$ direction, $k_{B}$ is the Boltzmann constant, $T$ the absolute temperature, $A=L_{x} \times L_{y}$ the area of the simulation box in the $x$ - $y$ plane, $r_{i j}$ the distance between two particles $i$ and $j$, and $U$ the TSF-LJ internal energy (with cut-off $\left.r_{c}^{*}=2.5 \sigma\right)$. The angular brackets denote a canonical average. For computational purposes, we slice the simulation box into a large number of slabs in the $z$ direction, where the width of each slab is set to $\Delta z \approx 0.1 \sigma$ (corresponding to 1141 slabs at $T^{*}=0.785$ and 1160 slabs at $T^{*}=0.855$ respectively). The surface tension is given by

$$
\gamma=\frac{1}{2} \int_{0}^{L_{z}}\left(P_{N}(z)-P_{T}(z)\right) d z
$$

The surface tensions that we obtain from MD simulations are $\gamma^{*}=0.204 \pm 0.007$ at $T^{*}=$ $0.785, P^{*}=0.026$ and $\gamma^{*}=0.098 \pm 0.008$ at $T^{*}=0.855, P^{*}=0.046$.

\section{References and Notes}

${ }^{1}$ Shusser, M.; Weihs, D. Int. J. Multiphas Flow 1999, 25(8), 1561-1573.

2 Brennen, C. E. Cavitation and Bubble Dynamics; Oxford University Press: New York, 1995. 
3 Suslick, K. S. Science 1990, 247, 1439-1445.

4 Dhir, V. K. Annu. Rev. Fluid Mech. 1998, 30, 156-159.

5 Volmer, M.; Weber, A. Z. Phys. Chem. 1926, 119, 277-301.

6 Farkas, L. Z. Phys. Chem. 1927, 125, 236-242.

7 Becker, R.; Döring, W. Ann. Phys. 1935, 24, 719-752.

8 Turnbull, D.; Fisher, J. C. J. Chem. Phys. 1949, 17, 71-73.

9 Kelton, K. F. Crystal Nucleation in Liquids and Glasses, in Solid State Physics, Ehrenreich, H. and Turnbull, D. Eds.; Academic Press: Boston, 1991.

10 Blander, M.; Katz, J. L. AIChe J. 1975, 21, 833-848.

11 Abraham, F. Homogeneous nucleation theory; Academic Press: New York, 1974.

12 Frenkel, J. Kinetic Theory of Liquids; Dover: New York, 1955.

13 Adams, G. W.; Schmidt, J. L.; Zalabsky, R. A. J. Chem. Phys. 1984, 11(81), 50745078.

14 Viisanen, Y.; Strey, R. J. Chem. Phys. 1994, 101(9), 7835-7843.

15 Hung, C.; Krasnopoler, M. J.; Katz, J. L. J. Chem. Phys. 1989, 90(3), 1856-1865.

16 Wagner, P. E.; Strey, R. J. Chem. Phys. 1984, 80(10), 5266-5275.

17 Rudek, M. M.; Katz, J. L.; Vidensky, I. V. J. Chem. Phys. 1999, 111(8), 3623-3629.

18 Lihavainen, H.; Viisanen, Y.; Kulmala, M. J. Chem. Phys. 2001, 114(22), 10031-10038.

19 Hruby, J.; Viisanen, Y.; Strey, R. J. Chem. Phys. 1996, 104(13), 5181-5187.

20 Luijten, C. C. M.; Baas, O. D. E.; van Dongen, M. E. H. J. Chem. Phys. 1997, 106(10), $4152-4156$.

21 Kim, Y. J.; Wyslouzil, B. E.; Wilemski, G.; W'olk, J.; Strey, R. J. Phys. Chem. A 2004, 108(20), 4365-4377. 
22 Gharibeh, M.; Kim, Y.; Dieregsweiler, U.; Wyslouzil, B. E.; Ghosh, D.; Strey, R. J. Chem. Phys. 2005, 122, 094512.

23 Wyslouzil, B. E., Strey, R., W'olk, J., Wilemski, G., Kim, Y., Eds. Nucleation and Atmospheric Aerosols, Proceedings of 17th International Conference; Springer: Netherlands, Galway, Ireland, 2007.

24 Sharaf, M. A.; Dobbins, R. A. J. Chem. Phys. 1982, r77(3), 1517-1526.

25 Scgmitt, J. L.; Zalabsky, R. A.; Adams, G. W. J. Chem. Phys. 1983, 79(9), 4496-4501.

26 Strey, R.; Wagner, P. E.; Schmeling, T. J. Chem. Phys. 1986, 84(4), 2325-2335.

27 Peters, F.; Paikert, B. J. Chem. Phys. 1982, 91(9), 5672-5678.

28 Herbert, E.; Balibar, S.; Caupin, F. Phys. Rev. E 2006, 74, 041603.

29 Caupin, F. Phys. Rev. E 2005, 71, 051605.

30 Zeng, X. C.; Oxtoby, D. W. J. Chem. Phys. 1991, 94(6), 4472-4478.

31 Oxtoby, D. W.; Talanquer, V.; Laaksonen, A. Annu. Rev. Phys. Chem. 1995, 46, $489-524$.

32 Kulmala, M., Wagner, P., Eds. Proceedings of the 14 th International Conference on Nucleation and Atmospheric Aerosols; Pergamon: New York, 1996.

33 Cahn, J. W.; Hilliard, J. E. J. Chem. Phys. 1958, 28(2), 258-266.

34 Cahn, J. W.; Hilliard, J. E. J. Chem. Phys. 1959, 31(3), 688-699.

35 Oxtoby, D. W.; Evans, R. J. Chem. Phys. 1988, 89(12), 7521-7530.

36 Balsara, N. P.; Lin, C.; Hammouda, B. Phys. Rev. Lett. 1996, r7(18), 3847-3850.

37 Pan, A. C.; Rappl, T. J.; Chandler, D.; Balsara, N. P. J. Phys. Chem. B 2006, 10(8), 3692-3696.

38 Delale, C. F.; Hruby, J.; Marsik, F. J. Chem. Phys. 2003, 118(2), 792-806. 
39 Shen, V. K.; Debenedetti, P. G. J. Chem. Phys. 1999, 111(8), 3581-3589.

40 Torrie, G. M.; Valleau, J. P. Chem. Phys. Lett. 1974, 28, 578-581.

41 van Duijneveld, J. S.; Frenkel, D. J. Chem. Phys. 1992, 96(6), 4655-4668.

42 Allen, R. J.; Warren, P. B.; ten Wolde, P. R. Phys. Rev. Lett. 2005, 94, 018104.

43 Allen, R. J.; Frenkel, D.; ten Wolde, P. R. J. Chem. Phys. 2006, 124, 024102.

44 Nosé, S. J. Chem. Phys. 1984, 81(1), 511-519.

45 Hoover, W. G. Phys. Rev. A 1985, 31, 1695-1697.

46 Frenkel, D.; Smit, B. Understanding Molecular Simulation: From Algorithms to Applications; Academic Press: Boston, 2nd ed., 2002.

47 Andersen, H. C. J. Chem. Phys. 1980, 72(4), 2384-2393.

48 Melchionna, S.; Ciccotti, G.; Holian, B. L. Mol. Phys. 1993, 78(3), 533-544.

49 Erringon, J. R.; Debenedetti, P. G.; Torquato, S. J. Chem. Phys. 2003, 118(5), 22562263 .

50 Lutsko, J. F. Phys. Rev. E 2006, 74, 021603.

51 Lutsko, J. F. private comunication 2008.

52 Wang, Z.-J.; Frenkel, D. J. Chem. Phys. 2005, 123, 154701.

53 ten Wolde, P. R.; Ruiz-Montero, M. J.; Frenkel, D. J. Chem. Phys. 1999, 110(3), 1591-1599.

54 Stillinger, F. H. J. Chem. Phys. 1963, 38(7), 1486-1494.

55 Nikunen, P.; Karttunen, M.; Vattulainen, I. Comp. Phys. Comm. 2003, 153, 407-423.

56 Bolhuis, P. G. J. Phys.: Condes. Matter 2003, 15, S113-S120.

57 ten Wolde, P. R.; Ruiz-Montero, M. J.; Frenkel, D. Faraday Discuss 1996, 104, 93-110. 
58 Sanz, E.; Valeriani, C.; Frenkel, D.; Dijkstra, M. Phys. Rev. Lett. 2007, 99, 055501.

59 Berne, B. J., Ciccotti, G., Coker, D. F., Eds. Classical and Quantum Dynamics in Condensed Phase Simulations; World Scientific: Singapore, 1997.

60 Ferrario, M., Ciccotti, G., Binder, K., Eds. Computer simulations in condensed matter: from materials to chemical biology, Lecture Notes in Physics; Springer-Verlag: Heidelberg, 2006.

61 Brenner, M. P.; Hilgenfeldt, S.; Lohse, D. Rev. Mod. Phys. 2002, 74, 425-484.

62 Potoff, J. J.; Panagiotopoulos, A. Z. J. Chem. Phys. 1998, 109(24), 10914-10920.

63 Smit, B. J. Chem. Phys. 1992, 96(11), 8639-8640.

64 Trokhymchuk, A.; Alejandre, J. J. Chem. Phys. 1999, 111(18), 8510-8523.

65 Wang, Z.-J.; Chen, M.; Guo, Z. Y.; Yang, C. Fluid Phase Equil. 2001, 183-184, 321329.

66 Walton, J. P. R. B.; Tildesley, D. J.; Rowlinson, J. S. Mol. Phys. 1983, 48(6), 1357 1368. 


\section{TABLES}

TABLE 1: Statistics collected from different sets of FFS "bubble-nucleation" trajectories at $T^{*}=0.855$ and $P^{*}=0.026$. $\left(W_{b}\right)_{i}$ denotes the order parameter at each interface $i$ as defined in section 2.2. $N_{i}^{s}$ is the total number of MD configurations (including momenta) collected at each interface $i$, irrespective of whether the bubble will continue to grow or it will collapse after interface $i$. $N_{i}^{p}$ is the average number of particles in the vapor phase inside or near the largest bubble. In this set, we include all particles that are inside or within $2.5 \sigma$ from the large bubble, identified using the approach described in section 2.2. From interface 14, bubble nearly always grows without collapse.

\begin{tabular}{|lccc|cccc|ccccc|cccc|ccc|}
\hline \hline$i$ & $\left(W_{b}\right)_{i}$ & $N_{i}^{s}$ & $N_{i}^{p}$ & $i$ & $\left(W_{b}\right)_{i}$ & $N_{i}^{s}$ & $N_{i}^{p}$ & $i$ & $\left(W_{b}\right)_{i}$ & $N_{i}^{s}$ & $N_{i}^{p}$ & $i$ & $\left(W_{b}\right)_{i}$ & $N_{i}^{s}$ & $N_{i}^{p}$ & $i$ & $\left(W_{b}\right)_{i}$ & $N_{i}^{s}$ & $N_{i}^{p}$ \\
\hline \hline 0 & 25 & 4012 & 50 & 3 & 92 & 1795 & 79 & 6 & 240 & 725 & 121 & 9 & 550 & 334 & 188 & 12 & 950 & 79 & 264 \\
\hline 1 & 37 & 3619 & 60 & 4 & 120 & 548 & 98 & 7 & 300 & 285 & 142 & 10 & 700 & 329 & 203 & 13 & 1200 & 147 & 271 \\
\hline 2 & 57 & 1304 & 70 & 5 & 180 & 512 & 103 & 8 & 400 & 320 & 170 & 11 & 820 & 374 & 215 & 14 & 1900 & 174 & 289 \\
\hline \hline
\end{tabular}




\section{FIGURE CAPTIONS}

Figure 1. Probability distribution of the number of neighbors $N$ for both the liquid and the vapor at coexistence $T^{*}=0.785$ and $P^{*}=0.026$. Two particles are neighbors if their distance is smaller than $r_{C}=1.6 \sigma$.

Figure 2. Diffusion coefficients as a function of time in the MD simulations with a Lowe-Andersen thermostat. The effect of $\nu^{*}$ on self-diffusion coefficient of the TSF-LJ fluid is shown.

Figure 3. MD simulation runs from the first to the second interface $\left(\nu^{*}=500\right)$ in a TSF-LJ system at $T^{*}=0.855$ and $P^{*}=0.026$.

Figure 4. Typical bubble-nucleation pathway in a super-heated liquid at $T^{*}=0.855$ and $P^{*}=$ 0.026. The top left frame shows the initial stage of the bubble, while the bottom right snapshot is the critical bubble.

Figure 5. Fluctuations of the order parameter as a function of MD time steps for a generic FFSinterface at $T^{*}=0.855$ and $P^{*}=0.026$. The jumps in $W_{b}$ are due to the merge and break-up of smaller bubbles.

Figure 6. Temperature at each interface from MD-FFS at $T^{*}=0.855$ and $P^{*}=0.026$. The green line denotes the temperature of the thermostat used in MD. The entire liquid bulk temperature is denoted with black solid square. The local temperature of the "both-backward-and-forward" largest bubble (blue open triangle) and the "only-forward" largest bubble (red solid circle) are also shown.

Figure 7. System volume at each interface from MD-FFS at $T^{*}=0.855$ and $P^{*}=0.026$. An expansion of the overall volume of the system is observable from interface 5 . 
Figure A-1. Comparison of temperature (left panel) and pressure (right panel) distributions between MD simulations and Gaussian distributions peaked at the same average value (continuous curves).

Figure B-1. $T-\rho$ liquid-vapor phase diagram. The filled squares denote the present simulation results of the TSF-LJ fluid $\left(r_{c}=2.5 \sigma\right)$. Errington's results for the same system are shown as a drawn curve. All other (open) symbols refer to simulation data for other variants of the LennardJones model (see text).

Figure C-1. Profiles of $\rho^{*}(z)$ (top panel), $P_{N}^{*}(z)$ and $P_{T}^{*}(z)$ (bottom panel, black-solid and red-dashed lines, respectively) of the liquid-vapor coexisting system at $T^{*}=0.785$ from MD simulations. We verified that the number densities of the liquid and vapor are the same as those of a saturated liquid and vapor at imposed temperature $\left(T^{*}=0.785\right)$, and that the normal pressure equals the saturated vapor pressure $P^{*}=0.026$ at this temperature. 


\section{FIGURES}

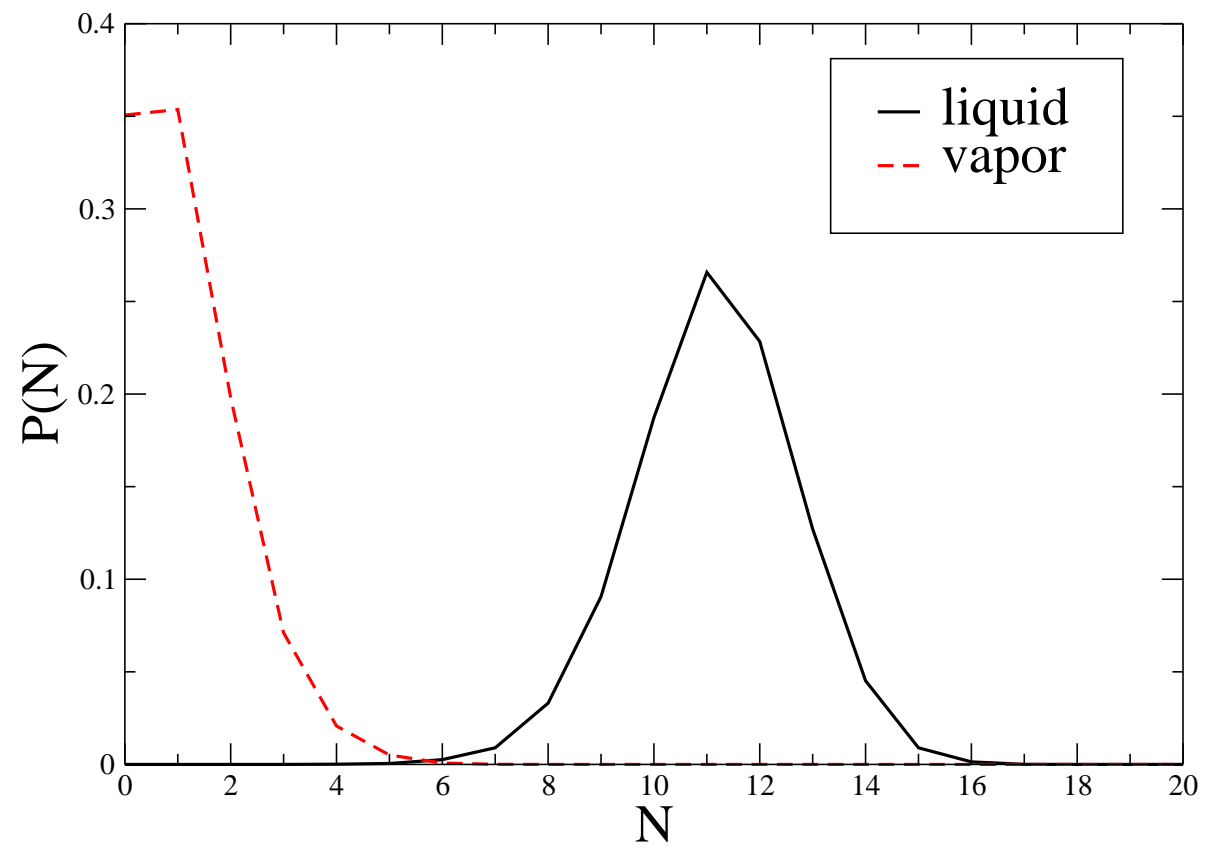

Figure 1 


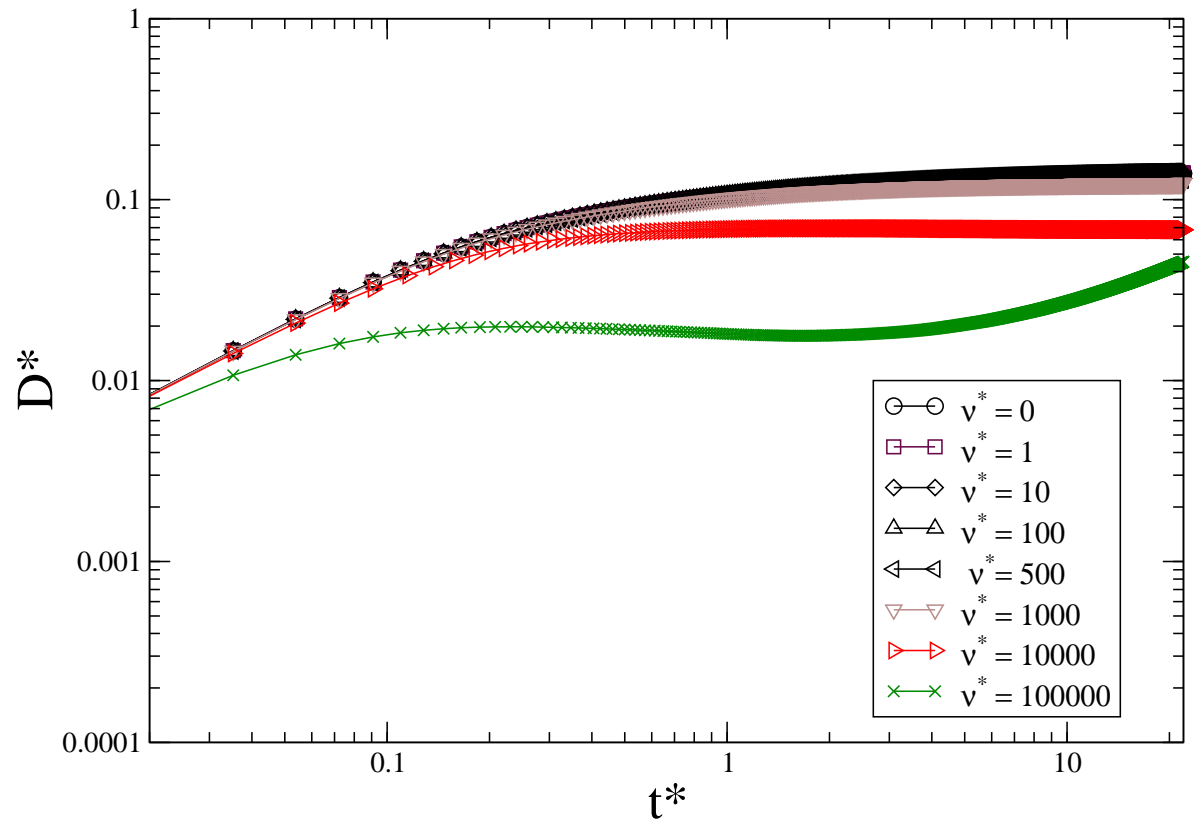

Figure 2 


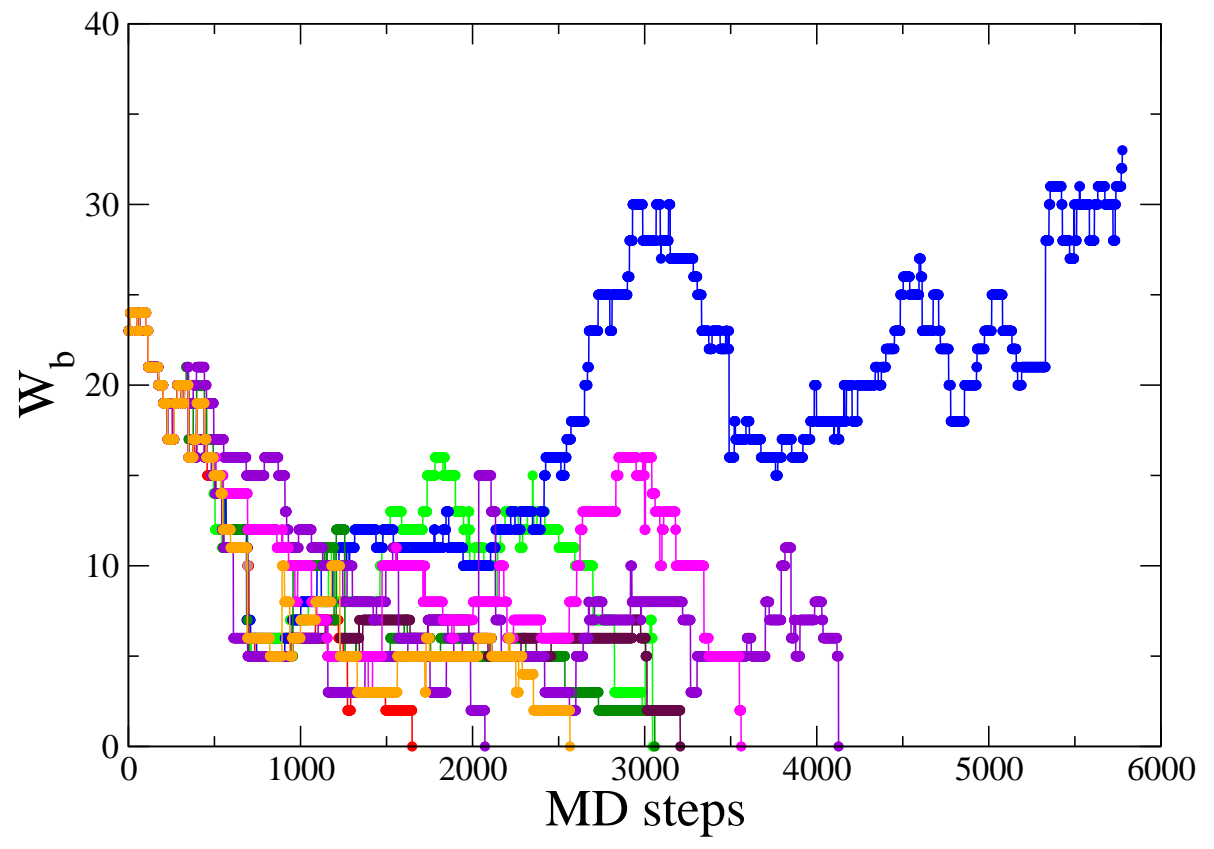

Figure 3 
0
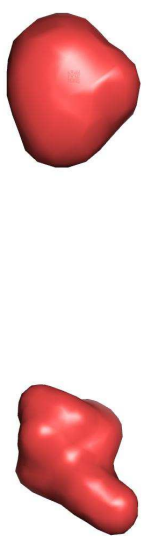

0
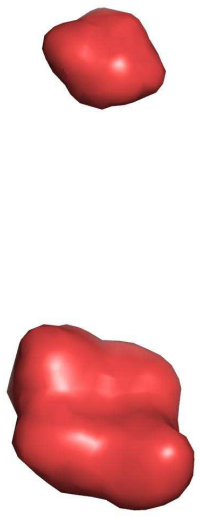

0
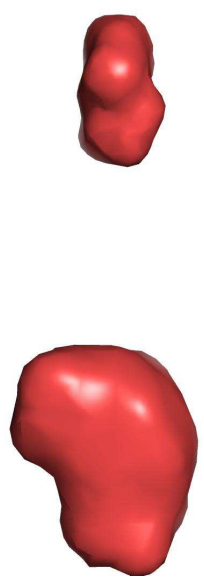

Figure 4 


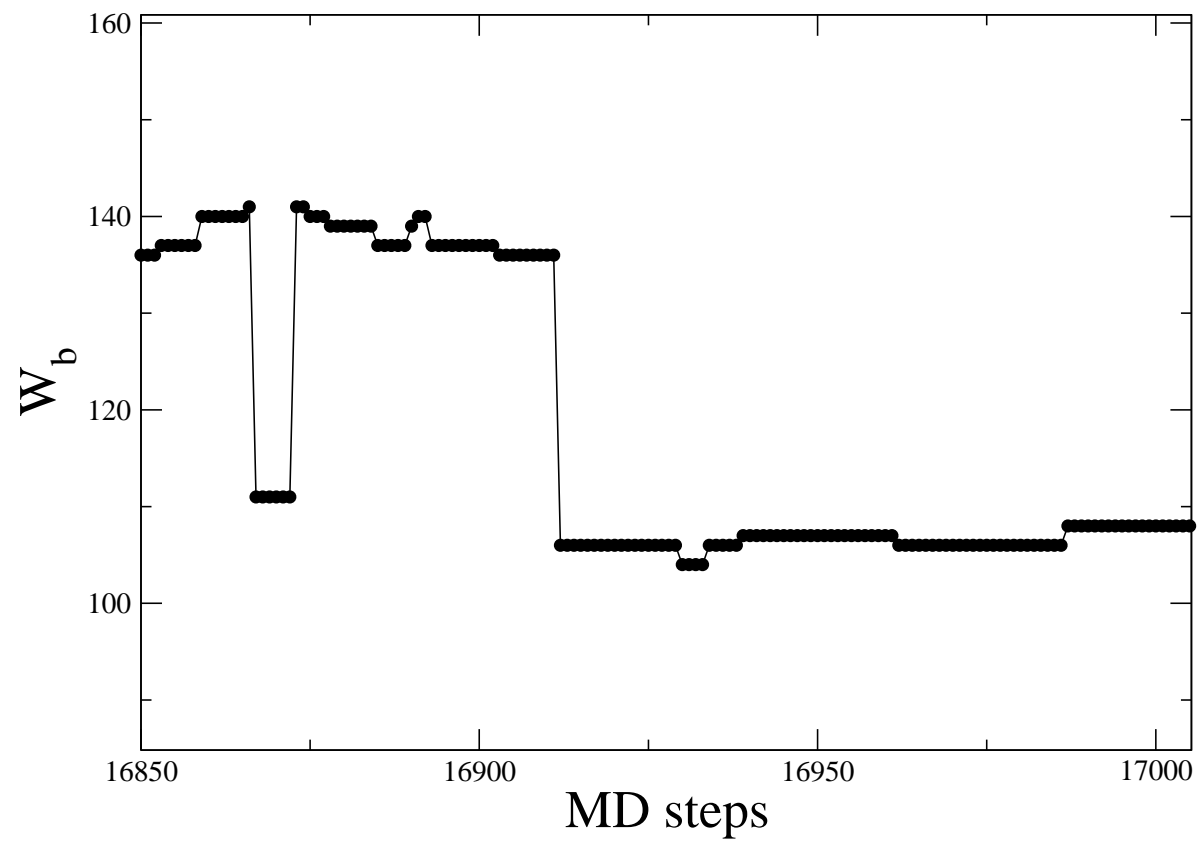

Figure 5 


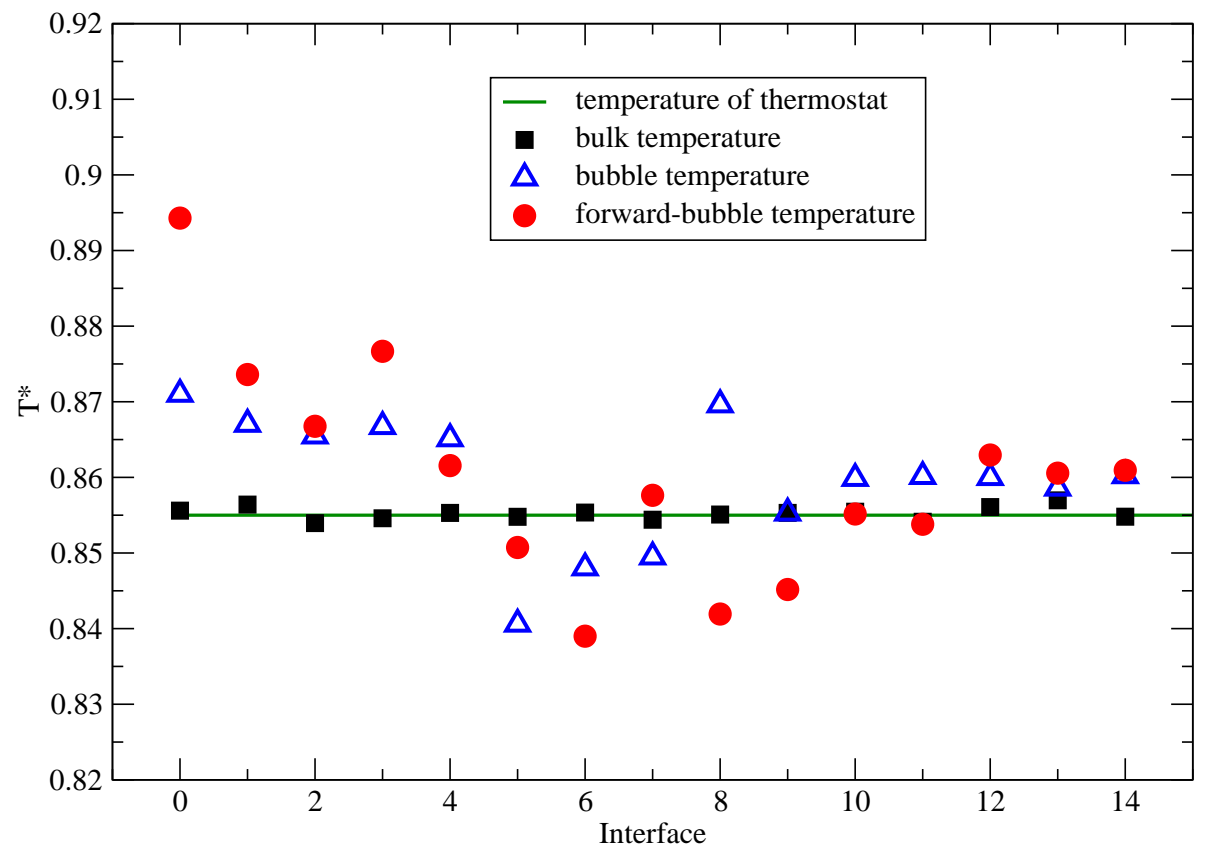

Figure 6 


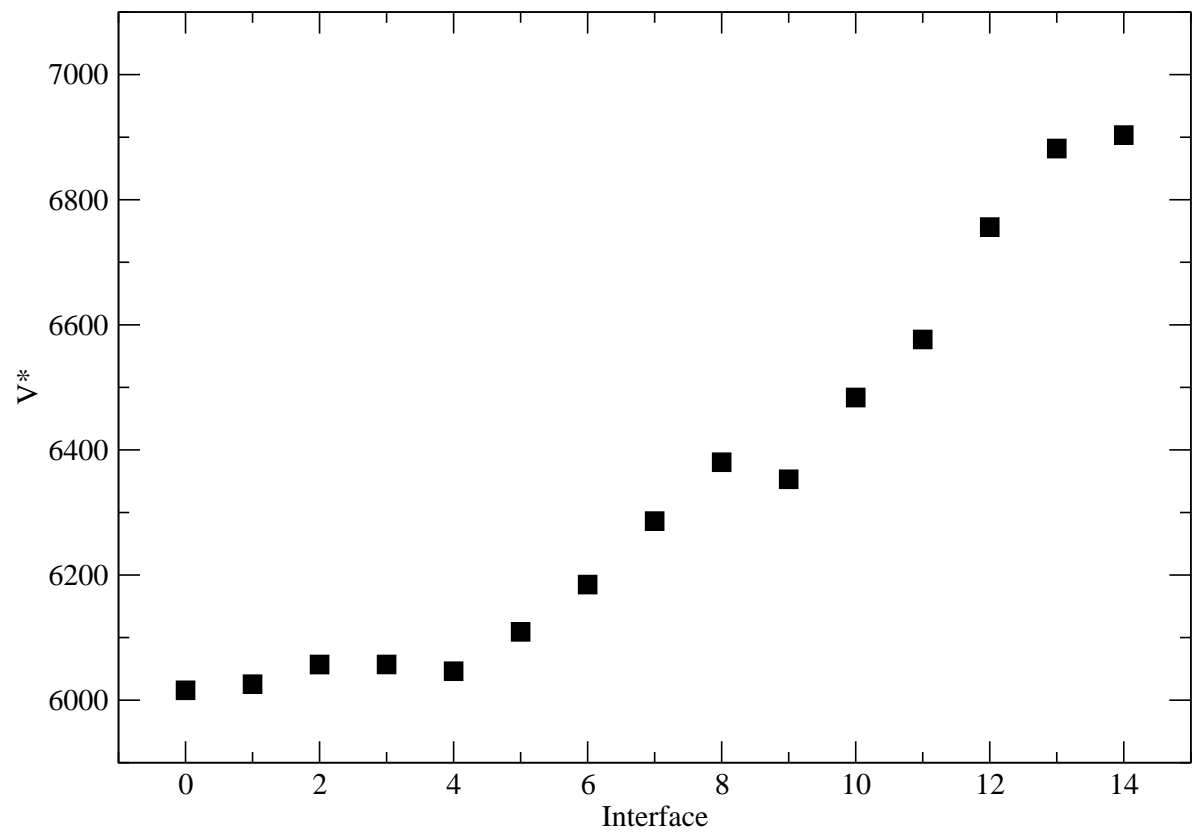

Figure 7 

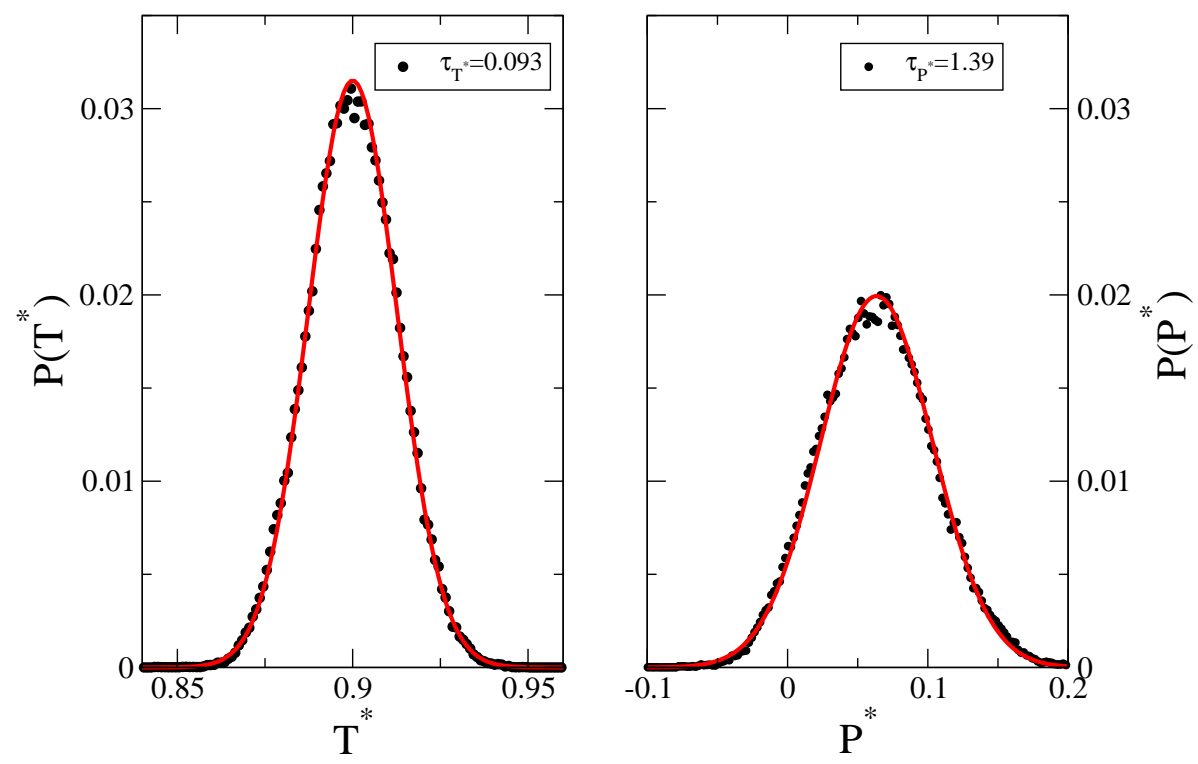

Figure A-1 


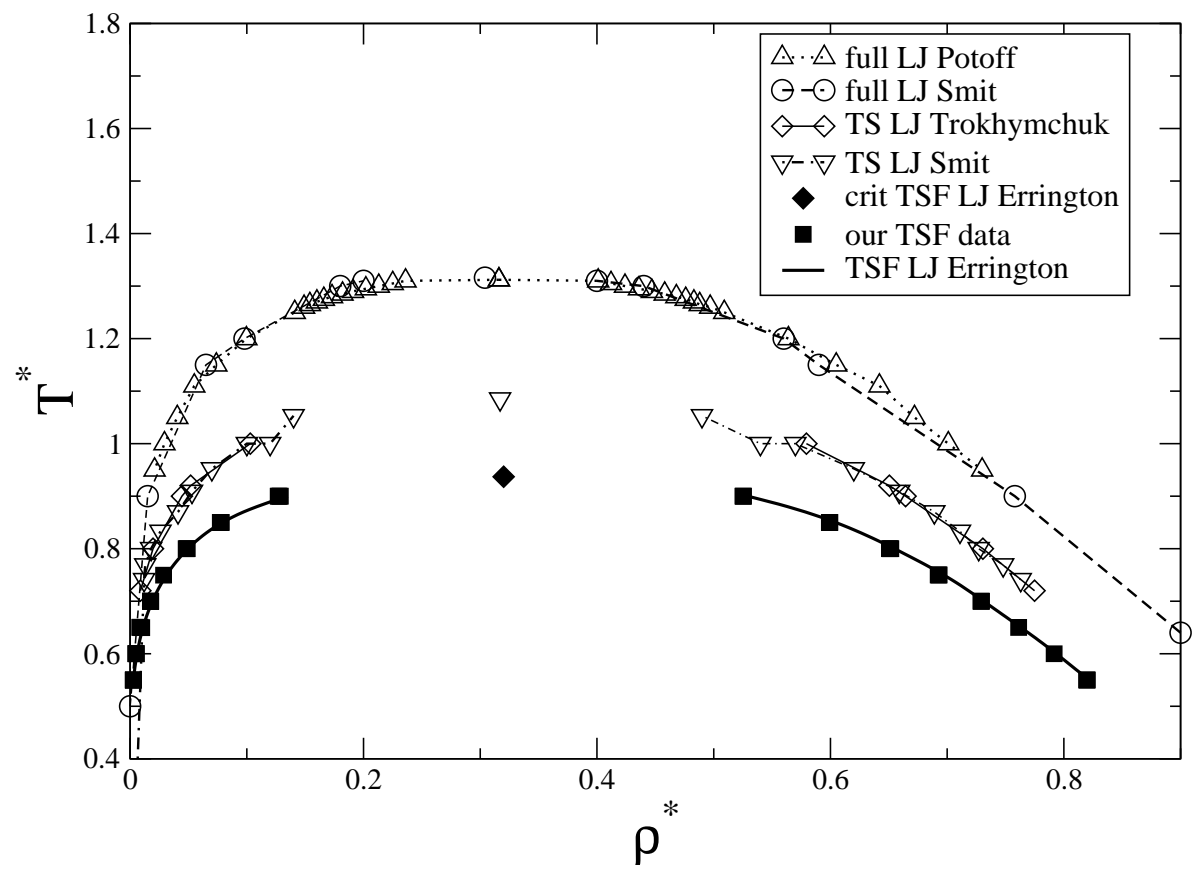

Figure B-1 

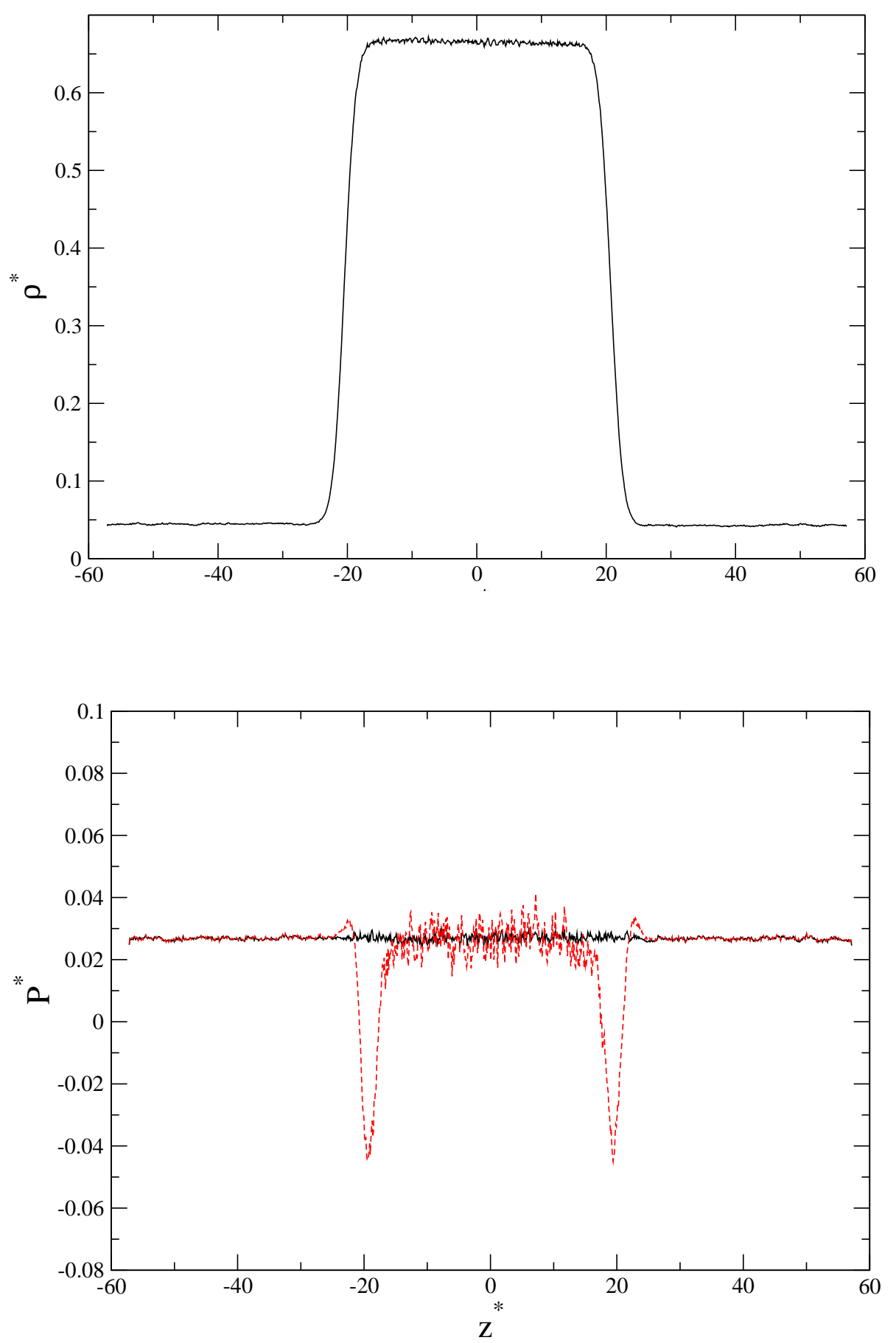

Figure C-1 